\title{
Encouraging Sustainable Fashion with a Playful Recycling System
}

\author{
Lilian Bosch \\ HKU, Utrecht School of the Arts \\ Oude Amersfoortseweg 131 \\ 1212 AA Hilversum, NL \\ Lilian.Bosch@student-kmt.hku.nl
}

\author{
Marije Kanis \\ HKU, Utrecht School of the Arts \\ Oude Amersfoortseweg 131 \\ 1212 AA Hilversum, NL \\ Marije.Kanis@kmt.hku.nl
}

\begin{abstract}
This paper presents the design and study of $\mathrm{UNI}^{2} \mathrm{BIN}$, a playful interactive system challenging its users to recycle their textiles so to adopt more sustainable fashion habits. The system initially rewards the user with direct playful interactions when depositing clothes in the recycling machine, followed by dispensing a token granting access to a user specific website further depicting what happens to their recycled clothes. To close the loop, a clothing line is created from the supplied textiles. This paper addresses the design of the system to encourage sustainable fashion and textile awareness amongst young adults, including the sustainability challenges, playful strategy used and the studies that informed the system, namely an interview study with 30 participants between 18 and 25 years old. Furthermore, preliminary lessons learned are presented from the design and evaluation of the system.
\end{abstract}

\section{Sustainability. Textile Recycling. Fashion. Persuasive design. Playful design}

\section{INTRODUCTION}

One of the biggest challenges for the global fashion industry is scarcity of resources, such as oil, water and land, as its demand is ever increasing [7]. At the same time, a tremendous amount of textile is being wasted: in The Netherlands alone, approximately 135 million kilos of textiles are wasted every year as ending up in landfills, while only about 70 million kilos is collected for recycling [11]. For sparing resources and taking pressure off landfills, recycling is an apparent solution for the fashion industry chain. As promising methods for better recycling are being developed -particularly upcycling techniques that can unravel and re-spin used fabrics into high-quality new fabrics- the collection of used textiles is becoming increasingly imperative. Thus, one of the things that the consumer can do to make a sustainable change is recycling their textiles instead of throwing them away. Consequently, it is important for people to become aware of this issue and to present durable ways that lead to a demand that would change the current fashion chain. However, research [14] suggests that particularly young adults' willingness to take action in helping the environment has been declining over the years. And thus, the question is how to positively motivate these people and engage them in lasting recycling and waste habits. For this purpose, this paper presents the iterative design of a playful textile recycling system (see
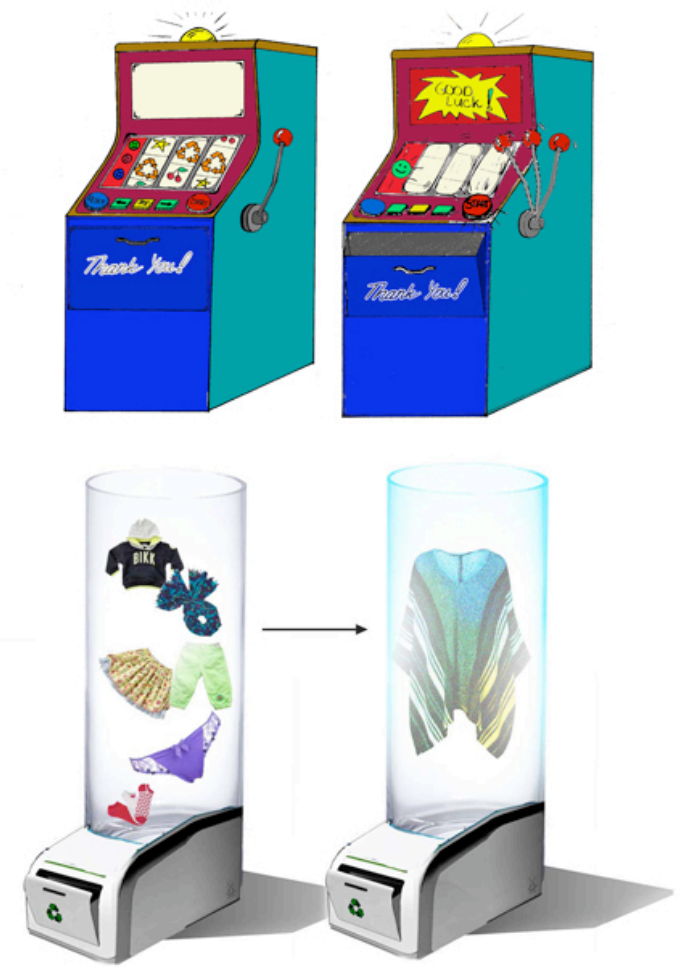

Figure 1: Initial sketches of the proposed playful recycling machine

Figure 1) that targets young adults. To engage the user in the entire recycling process, the work also 
includes a clothing line made out of the recycled fabrics, and a website that is linked to the system to keep the users informed about the process of what happens to their recycled clothes.

\section{RELATED WORK}

Environmental sustainability is a fast growing research field in $\mathrm{HCl}$. Approaching sustainability from the perspective of $\mathrm{HCl}$ [4] and sustainable Interaction Design (SID) [1, 2] is increasingly being explored. Indeed, these fields offer many insights in the design of systems that support environmental sustainable behaviour. Some researchers [8] have begun to conceptualize how fashion-related behaviour can become more sustainable through design, but more (practical examples of) digital solutions are needed to reach its full potential. The field of persuasive design [3, 12] offers an insight into what design strategies can be used to influence people to adopt more sustainable behaviour. For example, persuasive systems that use social influence to steer behaviour include the projects Wattsup? [5] and BinCam [12]. Wattsup? aims to motivate people to reduce domestic energy consumption using social networks. Like UNI ${ }^{2} \mathrm{BIN}$, BinCam aims to instigate users to reflect on recycling and waste habits. However, UNI ${ }^{2} \mathrm{BIN}$ focuses not only on the short duration of the disposal itself, but covers the whole recycling process instead.

An interactive installation that also aims to create lasting awareness of an environmental issue -in this case forest fires- is Play with fire [6], using a playful approach. Projects that regard waste recycling, such as The Bottle Bank Arcade Machine [9] and The World's Deepest Bin [10] by The fun Theory also show that a playful approach can be an effective way to encourage sustainable behaviour. UNI ${ }^{2} \mathrm{BIN}$ adopts a playful strategy as well, but focuses on the less investigated environmental issue of textile recycling.

\section{DESIGN STUDIES}

Considering related work, the large amount of textiles being wasted and the prospect of making high-quality clothes out of recycled ones, the design concept was based on a system to engage young adults in the recycling process. To inform the design of such a playful system for promoting textile recycling, several design studies were conducted, namely a short recycling experiment and a lengthier interview study to evaluate young adults' attitudes towards recycling textiles. Furthermore, small-scale studies with design iterations were carried out. Lastly, the final recycling machine was evaluated to study how users interacted with and understood the system.
The studies were all conducted at a School for Arts, Media \& Technology so that young adults between 18 and 25 years could be targeted at one location.

\subsection{Recycling experiment}

The first stage of the study was a recycling experiment for which an email was sent out to approximately 1100 students of the same university (Utrecht school of the Arts). It concerned a call to all students to bring as many textiles as possible (including worn down and torn textiles) to the school's location at a specific moment and recycle them through a provided recycling bank in exchange for a reward. Participants could choose between something 'meaningful', which meant an extensive thank-you letter explaining the positive value of their actions, or something 'yummy': a cookie. The email was sent out one day prior to the test and took place in the school's canteen for a period of two hours. This experiment was conducted for mainly three purposes: (i) Explore initial recycling behaviour without intervention of a playful system; (ii) Investigate what kind of reward would motivate recycling, and (iii) Gain and collect items that could be used as (inspirational) material for the design of a fashion line.

\subsubsection{Findings from the recycle experiment}

Only nine students responded to the email call and brought clothes to recycle, as shown in Figure 2.

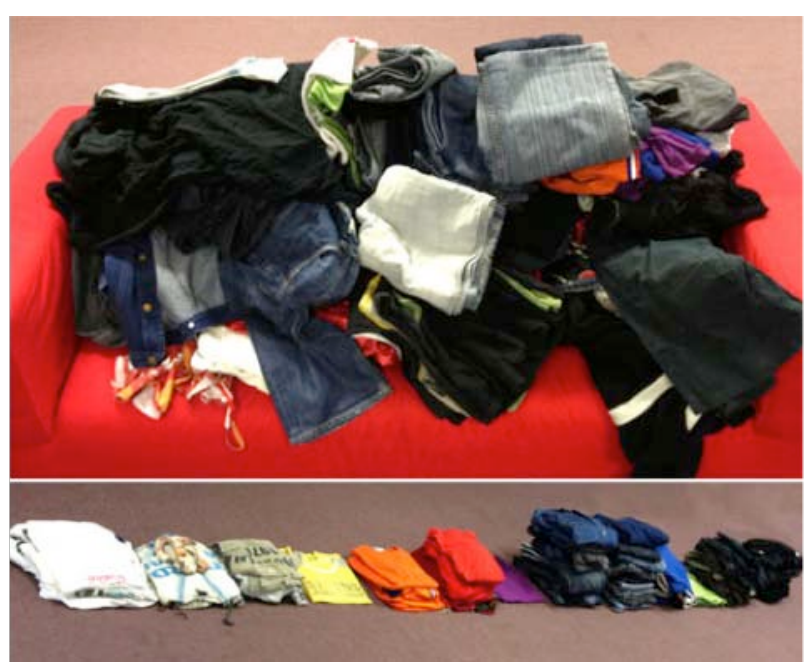

Figure 2: Some of the collected textiles from the recycling experiment.

The amount of contributed clothes per participant could be considered high: nine students contributed around four large garbage bags in total that were filled with textiles. Jeans were the most collected type of garment. Three participants (33\%) did not want a reward, but only wanted to dispose their brought textiles. The 'meaningful' reward was most favoured as this was chosen by the other six participants (67\%) and positively valued by all. 


\subsection{Semi-structured interview study}

The first brief call to recycle led to only few participants. Therefore, semi-structured interviews were held to: (i) Reach a larger group of participants, and (ii) Learn more about the young adults' general recycling habits. Participants were targeted randomly within the school's grounds. Questions regarded their current fashion consumption and textile disposal, and general recycling knowledge and perceptions.

\subsubsection{Results from the interviews}

The interviews were held with 30 participants, from which 18 were male and 12 were female. The interview results suggest that participants were often unaware of more sustainable options that they could make, leading to textiles unnecessarily wasted. Interestingly, only a small minority of the participants (10\%) associated the purpose of recycling textiles with environmental benefits. The majority of the participants $(60 \%)$ associated the purpose of recycling textiles solely with secondhand use. As one of the participants stated:

\begin{abstract}
"Recycling textiles is valuable for helping the poor. When my garments no longer look nice or have holes, I throw them away. I don't see the point of recycling something that is already worn down."
\end{abstract}

Half of the participants (50\%) indicated to normally throwaway textiles for such similar reasons. Notably, almost all of the participants $(93 \%)$ stated to regularly recycle other types of waste, such as glass and paper, which they associated with protecting the environment. It was considered difficult by most of the participants to describe how sustainable their clothes are. Longevity was the most popular mentioned reason by ten participants $(33 \%)$ for perceiving their clothes as sustainable. Only one participant (3\%) indicated that a garment's material played a part in deciding whether or not her clothes were sustainable. She stated:

\footnotetext{
"My most sustainable garment is my sweater. It's made of $100 \%$ wool, so that must be very natural and sustainable material. [...] I don't think about sustainability when buying clothes. Then again, I don't feel that I am provided with a choice. I really have no idea which garment is sustainable and which one isn't."
}

None of the young adults were aware of the possibilities to recycle textiles for making new fabrics or other products. Upcycling, the process of converting old or discarded materials, such as textiles into something of better use or higher quality was also an unfamiliar concept to them. However, the majority of the participants $(77 \%)$ indicated to be interested in finding out more. Therefore, it was found that the proposed system should make the target group more aware of the latent potential and added value of textile recycling.

\subsection{Design iterations}

Based on the previously described studies and discussions with collaborative experts in the field of sustainable fashion, several iterations of a playful textile-recycling machine were designed (such as Figure 1, 3, and 4). Some were early dismissed in the design process, such as a hologram machine (Figure 3), because of reasons as impractical lightning and scaling conditions.

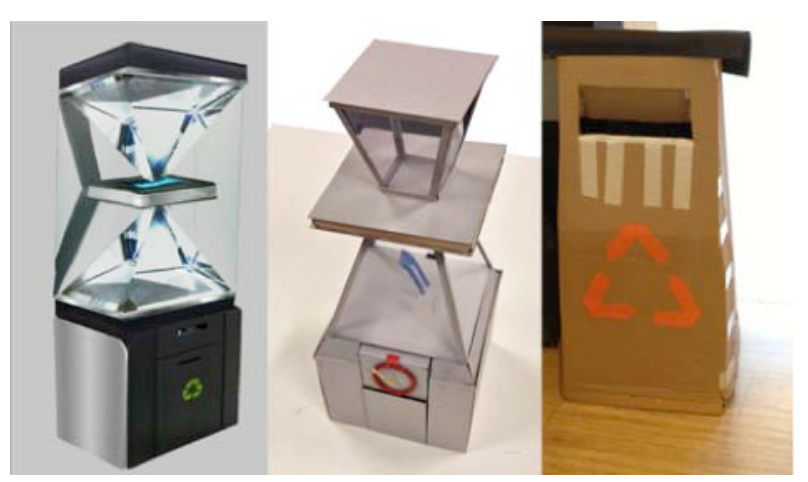

Figure 3: Iterative designs of the recycle bank: card-box prototype (right) \& hologram machine (left, centre)

Another prototype included a simple card-box version, which was used to evaluate how users responded to different types of rewards -such as confetti coming out of the bin's opening and music playing- and another textile recycling bin which used balloons representing oil, co2 and water to inform the user about environmental resources saved by recycling textiles (Figure 4).
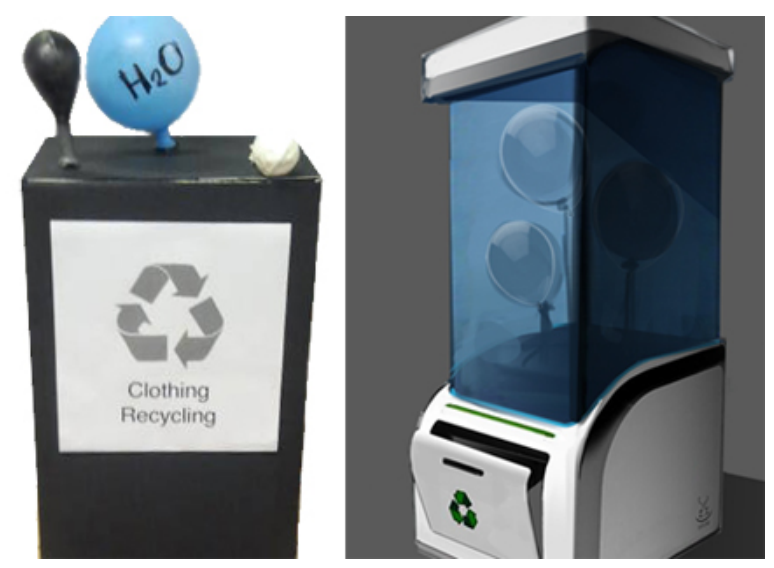

Figure 4: The balloon prototype: card-box prototype (left) and sketch iteration (right)

These prototypes were consecutively placed and tested in the school's canteen for few hours to gauge initial reactions. Students that passed-by were observed or asked to use these prototypes. For the interactive card-box prototype, this involved 
15 passers-by, and for the balloon prototype, 12 participants interacted with this.

\subsubsection{Findings iterative design}

Several conclusions were drawn from the evaluation of these prototypes: (i) The young adults responded positively to the containers' unexpected feedback. As soon as the container did something they were not expecting, it got their full attention. (ii) Terms such as oil, co2 and amounts of water in relation to how much of these resources were saved by recycling textiles were not directly valued by the passers-by. Therefore, for our final design, we believed that such data should be easier to directly identify with. Furthermore, as participants seemed to positively respond to unexpected feedback, for the final design of the system we believed that unexpected, surprising interactions, revealing the mysterious recycle process should play a leading role.

\section{SYSTEM DESIGN: UNI²BIN}

Informed by the initial studies and iterative prototypes, the interactive system UNI ${ }^{2}$ BIN (derived meaning: you need to - bin) was designed by a multi-disciplinary team (including textile and fashion sustainability experts, game-, interaction- and fashion designers) with the aim to increase awareness of the beneficial aspects of recycling textiles into new fabrics. The final interactive recycling machine (Figure 5,8 ) provides direct information about what happens to the clothes when these are recycled via short animations that can be seen by the user and bystanders through various peepholes that are spread over its surface.

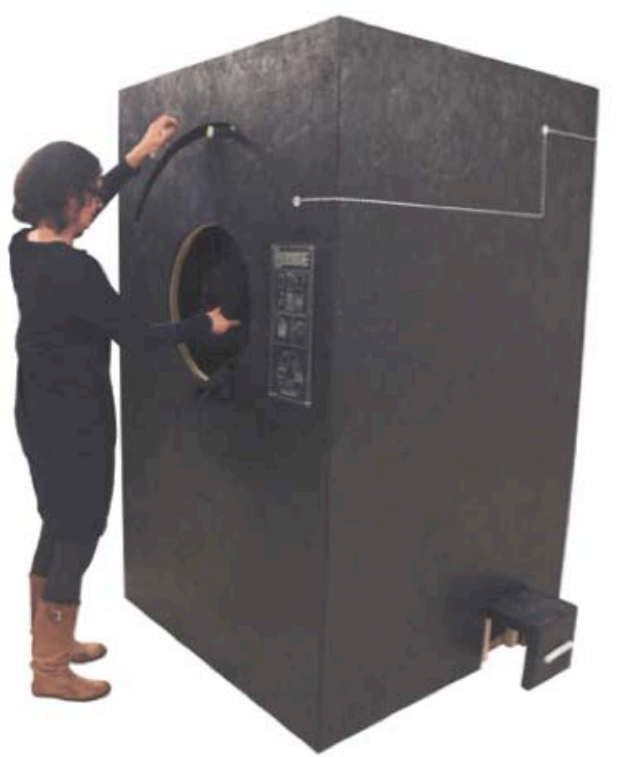

Figure 5: A user interacting with the final recycling machine.

The machine is about average size for a textile recycle bin $\left(1.2 \times 1.2 \times 2.0=2,9 \mathrm{~m}^{3}\right)$ and contains room for the deposited textiles, a computer, four screens (one for each peephole), two speakers, a printer for dispensing tickets, and custom built softand hardware to start the machine upon interaction with the mechanical aperture. As soon as textiles have been interactively disposed in the machine's aperture, sounds and animations of the textile recycling and production processes start to play. The machine then disposes a ticket that links to a website enabling users to track their own contributions. To close the loop, a clothing line has been designed out of the recycled fabrics.

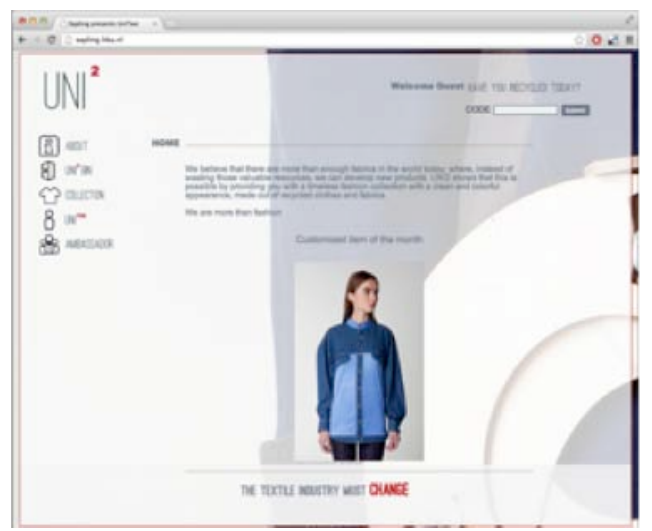

Figure 6: The $U N I^{2} B I N$ website

The website, as shown in Figure 6, was designed to (a) be able to reach and engage users for a longer period than just the short moment of using the textile-recycling machine; (b) enable updates and additions to further encourage sustainable behaviour, e.g. via playful challenges as "wash your clothes at 30 instead of 40 degrees" and so help to create longer lasting sustainable lifestyles; (c) let users keep track of and reflect upon their contributions in recycling and suggest sustainable alternatives; (d) present the clothing line (which users contribute to through recycling their textiles) (Figure 7).

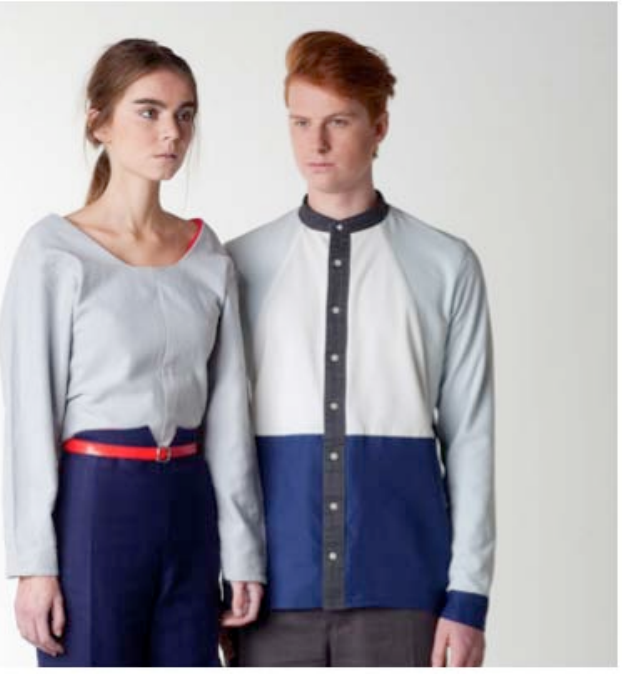

Figure 7: The clothing line 
The current fashion line has been created out of recycled material that was acquired through the design study. This also included a visit to Humana, a large distribution and sorting organisation for recycled textiles, to acquire additional inspiration and material. The line has been created to set a tangible example for using recycled fabrics to create new garments, and as a reward to all the people who contributed their old textiles to the program. The inside of each item contains instructions on what to do with it after use: recycle it again.

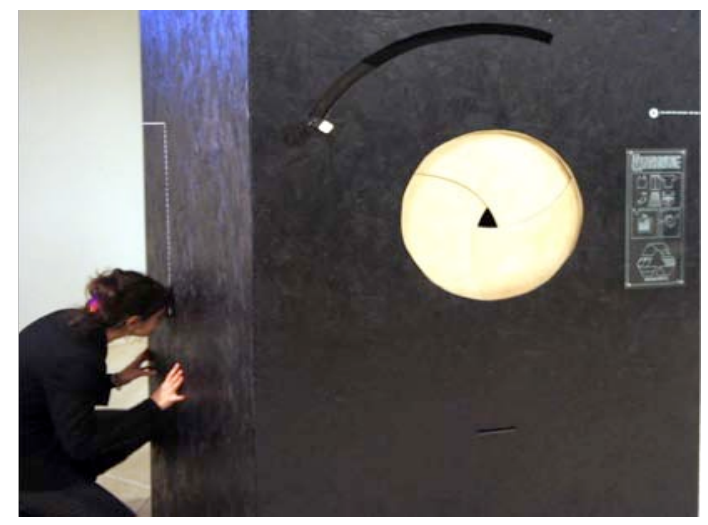

Figure 8: A user looking at one of the animations inside a peek hole of the recycling machine

\section{EVALUATION OF THE FINAL SYSTEM}

A user study was conducted with the final, functional version of the UNI ${ }^{2} \mathrm{BIN}$ (Figure 5, 8) that dispenses a ticket after each use, referring the participant to the website. The study lasted one week and included 28 participants in total in which the final machine was located in one of the university's hallways. Students that happened to pass by the system were asked to participate in a short user study. Machine usage was observed from a short distance and field notes were taken. After the user test was complete, the participants were asked to fill out a survey on a provided computer. Questions regarded their overall experience with the machine, which kind of wordings they associated with the experience, whether they planned on visiting the website and whether they felt more motivated about recycling textiles than prior to the test.

\subsection{Findings from the user study}

The sounds that start playing directly after disposing clothes encouraged its users to take a closer look at the machine, often resulting in the discovery of the peek holes and positively responding to the animations shown. The results from the survey indicated the following: from a total of 28 participants, a majority $(57 \%)$ indicated to associate and experience the playful system as 'weird and wonderful'. Some users (39\%) indicated to believe that their clothes were being renovated inside the machine. Half of the participants $(50 \%)$ indicated to feel more motivated towards recycling their old textiles than prior to the study.

The findings from the user study further suggest that the system made the participants feel more lastingly engaged in the whole textile recycling process. As one of the participants stated:

\footnotetext{
"It is interesting for me to still be involved even after having disposed your clothes. Seeing what will happen to these makes me hungry for more information."
}

In this vein, almost half of the participants (46\%) stated in the survey that they intended to use the ticket and website to find out more. In addition to the test cases, many passersby were observed to show interest in interacting with the machine by looking into the peek holes or opening and closing its aperture to see what would happen.

\section{LESSONS LEARNED}

Although more studies are needed to evaluate the effectiveness of the playful recycling system, the preliminary lessons learned from this study are the following:

- Recycling textiles is a promising, but neglected solution to sparing resources in the textile industry.

- Recycling textiles seems to be highly associated with second-hand use by the young adults involved and not with upcycling or protecting the environment. Playful design and interactive solutions can help to broaden this perspective to prevent textiles and scarce resources being wasted.

- The participants were often unaware of the more sustainable options that they could make. Playful interactive interventions can help to inform, engage and address this.

- More attention is needed in the $\mathrm{HCl}$ area to design and create playful digital examples that motivate lasting sustainable action.

- To motivate change, it seems important to present the positive effects of the desired sustainable behaviour in ways that are meaningful, transparent and easy to relate to.

- A playful approach with a sense of mystery and extraordinary feedback can help to attract people and seduce them to adopt and engage in more sustainable behaviour.

- Multifaceted issues need a broad, multidisciplinary approach. 


\section{DISCUSSION AND FUTURE WORK}

This paper described the iterative design process of the UNI ${ }^{2} \mathrm{BIN}$ recycling system, as an example for motivating and engaging young adults in lasting sustainable textile recycling habits. A playful strategy was particularly explored to motivate sustainable action. The UNI ${ }^{2} B I N$ system informs its users about the value of recycling, by showing that new textiles and garments can be made out of their old textiles. It aims to change the biases around textile recycling being only about second-hand use and to make people aware of the true need to recycle. The results of this work support other work (e.g. [6]) concerning the effective use of playful approaches to persuade sustainable behaviour. $\mathrm{UNI}{ }^{2} \mathrm{BIN}$ seemed to be effective in motivating users to recycle. However, the studies were of a modest scale and the over-all design needs to be further optimized, evaluated, advanced and positioned in the whole fashion and textile recycling chain. Next studies should include an evaluation of the system for a longer period of time with a larger public and a comparative study of other (non) playful strategies. This would enable more insights of the effectiveness of the system, how such recycle system and website would be used over a longer period of time and how systems can be best designed for young adults and a whole community to motivate sustainable behaviour. The ultimate goal is to shift the main use of virgin fabrics in the fashion industry to upcycled fabrics, making this the new standard.

\section{ACKNOWLEDGEMENTS}

We thank the study participants and Humana. We acknowledge Hanne Marckmann, Marina Toeters, Sanne van der Wal, Jethro Jongeneel, Thom Wouters, Hiromi Haneda, Charly Cheung, Minghan Tsai, Michiel Deen, Esmay Hijmans, Bob Gussenhoven, Aljona Minejeva, Anbasja Blanken, Angela Groen, Evelyn Grooten, Marinka Copier, Ellen Sillekens and Anita de Wit for their considerable contributions to this work.

\section{REFERENCES}

1. Blevis, E. (2006) Advancing sustainable interaction design: Two perspectives on material effects. Design philosophy papers, 4.

2. Blevis, E. (2007) Sustainable interaction design: Invention \& disposal, renewal \& reuse. Proceedings of CHI'07. San Jose, California, USA: ACM, 503-512.
3. Comber, R. \& Thieme, A. (2012) Designing beyond habit: Opening space for improved recycling and food waste behaviors through processes of persuasion, social influence and aversive affect. Personal and Ubiquitous Computing, 1-14.

4. Disalvo, C., Sengers, P. \& Brynjarsdóttir, H. (2010) Mapping the landscape of sustainable $\mathrm{HCl}$. Proceedings of CHI'10. Atlanta, Georgia, USA: ACM, 1975-1984.

5. Foster, D., Lawson, S., Blythe, M. \& Cairns, P., (2010) Wattsup?: Motivating reductions in domestic energy consumption using social networks. Proceedings of NordiCHI 2010. Reykjavik, Iceland: ACM, 178-187.

6. Mendes, M., Ângelo, P., Nisi, V. \& Correia, N. (2011) Play with fire. Proceedings of $A C E^{\prime} 11$. Lisbon, Portugal: ACM.

7. Mundinger, A.K. (2009) Resource scarcity and textile production ZTC Working Paper, VDI Technologiezentrum, Düsseldorf.

8. Pan, Y., Roedl, D., Thomas, J.C. \& Blevis, E. (2012) Re-conceptualizing fashion in sustainable $\mathrm{HCl}$. Proceedings of DIS'12. Newcastle, United Kingdom: ACM, 621-630.

9. The fun theory (2009a) The bottle bank arcade machine, http://www.thefuntheory.com/bottlebank-arcade-machine, (Retrieved May 23, 2013).

10. The fun theory (2009b) The world's deepest bin http://www.thefuntheory.com/worlds-deepestbin (Retrieved May 23, 2013).

11. Schalkwijk, M. and Mulder, S. (2012) Kansen voor textielinzameling (Opportunities for Textile collection), TNS-nipo, Amsterdam.

12. Thieme, A., Comber, R., Miebach, J., Weeden, J., Kraemer, N., Lawson, S. \& Olivier, P. (2012) "We've bin watching you": Designing for reflection and social persuasion to promote sustainable lifestyles. Proceedings of $\mathrm{CHI}^{\prime} 12$. Austin, Texas, USA: ACM, 2337-2346.

13. Tscheligi, M. \& Reitberger, W. (2007) Persuasion as an ingredient of societal interfaces. interactions, 14 (5), 41-43.

14. Twenge, J., Freeman, E. and Campbell, W. (2012) Generational differences in young adults' life goals, concern for others, and civic orientation, 1966-2009. Journal of Personality and Social Psychology, 102 (5), 1045-1062. 